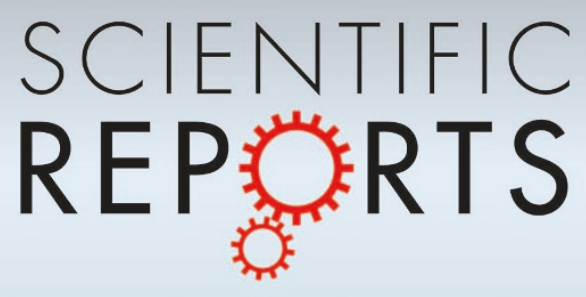

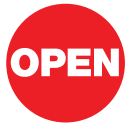

SUBJECT AREAS:

PHASE TRANSITIONS

AND CRITICAL

PHENOMENA

NONLINEAR PHENOMENA

STATISTICAL PHYSICS

COMPLEX NETWORKS

Received

10 December 2012

Accepted

19 February 2013

Published

4 March 2013

Correspondence and requests for materials should be addressed to

M.T. (mat51@phy. duke.edu)

\section{Role of committed minorities in times of} crisis

\author{
Malgorzata Turalska', Bruce J. West ${ }^{1,2}$ \& Paolo Grigolini ${ }^{3}$ \\ 'Department of Physics, Duke University, Durham, NC, ${ }^{2}$ Information Science Directorate, Army Research Office, Durham, NC, \\ ${ }^{3}$ Center for Nonlinear Science, University of North Texas, Denton, TX.
}

The surprising social phenomena of the Arab Spring and the Occupy Wall Street movement posit the question of whether the active role of committed groups may produce political changes of significant importance. Under what conditions are the convictions of a minority going to dominate the future direction of a society? We address this question with the help of a Cooperative Decision Making model (CDMM) which has been shown to generate consensus through a phase-transition process. We observe that in a system of a finite size the global consensus state is not permanent and times of crisis occur when there is an ambiguity concerning a given social issue. The correlation function within the cooperative system becomes similarly extended as it is observed at criticality. This combination of independence (free will) and long-range correlation makes it possible for very small but committed minorities to produce substantial changes in social consensus.

ocial progress and change can be portrayed from at least two competing perspectives. The first is that of a gradual, cumulative evolution of thoughts and opinions providing a smooth gradual transition from one orientation to another. The second, more contemporary view approaches history as a series of status quo periods interrupted by abrupt, intermittent and sometimes violent events ${ }^{1}$. Those brief moments of social unrest often bring an opportunity for radical change within the structure of society or its political organization. As the events of the last century, such as the Velvet revolution, the fall of the Berlin wall and the Solidarity movement in Poland, have demonstrated, the instances of social unrest can lead to the replacement of totalitarian regimes by democratic parliamentary systems. However, equally often the transformation proceeds in the opposite direction, where one dictator is replaced by another, as happened during the Cuban revolution and in many African countries in the 1960 's. In extreme cases unrest can lead to political destabilization, creating an environment in which extremist or terrorist ideas thrive. As a result of globalization, social and political changes that occur in one place in the world almost immediately have a wider almost universal effect. The interdependence of economies, cultures and organizations create a complex network in which even weak interactions can have a profound effect on the spread of ideas and opinions. Therefore the understanding of what conditions have to be realized for a given "movement for change" to succeed is an issue of overwhelming importance 2 .

Recent studies demonstrate that the abrupt, discontinuous events such as the catastrophic failure of power grids, computer networks and financial market crashes are a consequence of the high connectivity of those networks ${ }^{3}$. Therefore it is important to realize that the local interactions between the building blocks of such networks play a role analogous to that of the interactions between particles in solid state matter, which suggests that adopting a phase-transition perspective to describe complex network dynamics ${ }^{4}$ is a natural application of statistical physics to network science. Thus, in a manner analogous to the abrupt phase transitions seen in physical phenomena, social and political dynamic discontinuities arise from the local interactions among the elements of those systems and result in a tipping point. Consequently the cooperative behaviour within social groups need not be caused by external factors but can be determined by internal interactions.

As early as 1975 Haken $^{5}$ used the phase transition concept to interpret the 1968 French student revolution. His approach explained the remarkably rapid transition from traditional morality to sexual liberation, but he did not investigate the role the minority of protesting students may have played in triggering this change. Only recently the concept of inflexible agents (committed minorities) who retain their opinion regardless of their social environment has been introduced by Galam and $\mathrm{Jacob}^{6}$. These minorities are known as zealots in the sociology literature. Masuda ${ }^{7}$ investigated the influence of zealots on cooperation in evolutionary phenomena using social dilemma game theory. He found that even a small fraction of zealous co-operators results in a large fraction of cooperation in the evolutionary dynamics. Mobilia et al. ${ }^{8}$ determined, using a master equation form of the voter 
model, that zealots balanced between parities can also inhibit consensus even when there is a robust majority of one party over another.

Xie et al. ${ }^{9}$ studied the influence of these inflexible individuals using a Naming Game approach and found that when this committed minority reaches a threshold of $10 \%$ of the population the opinion of the entire social network can be reversed to conform to that of the minority. This result was shown to be largely independent of the structure of the model interactions within society but can be determined by as much as $10 \%$ to as little as $4 \%$ for a sparse network ${ }^{10}$. The percentage at which the tipping point (critical point of a phase transition) occurs is clearly model dependent and can vary from 4 to $15 \%^{11,12}$.

Even more interestingly, Xie et al. ${ }^{7}$ make the observation that the onset of the American civil rights movement overlapped with the African-American approaching $10 \%$ of the United States population, and suggested historic relevance for their numerical investigations. More recently, Couzin et al. ${ }^{13}$ presented experimental evidence that a committed minority of fish can override the consensus of a substantially larger school of fish, reintroducing variability of opinions within the system.

Social models provide a rich dynamics that is associated with the nature of the interactions among the members of the network, as well as the changing structure of the network itself. Singh et al. ${ }^{14}$ investigated how these two aspects of the dynamics influence the time to reach consensus as a function of network size $\mathrm{N}$. They found a critical value of the committed fraction. The time to reach consensus in the subcritical domain is exponent in network size and transitions to logarithmic in network size in the supercritical domain. The critical minority fraction is determined to be $10 \%$.

Herein we further investigate the conditions under which committed minorities can initiate global changes. With the use of a previously developed Cooperative Decision Making Model (CDMM) $1^{15,16}$ we demonstrate that the abrupt changes in the organization of social groups, rather than being moments of disorder, are instances of increased spatial correlation between the units of the network. This condition of extended cooperation, like that of the critical state of a phase transition, allows for a small subgroup of the society to exert remarkable influence over the whole system. In the results section we briefly review some material that has previously been published, but which is necessary to formulate a clear understanding of what is entailed by CDMM dynamics. The microscopic dynamics of the two-state master equation is replaced by a stochastic macroscopic global variable that is determined to undergo a phase transition ${ }^{16}$. The results in the sections on the scaling of critical exponents, the three-dimensional CDMM, the correlation function and the committed minority are new and show by direct calculation that the CDMM dynamics belong to the Ising universality class. This last result is consistent with a general claim made by Grinstein et al. ${ }^{17}$ that universality occurs for a large class of two-state probabilistic lattice models with local interactions as pointed out to us by an anonymous referee.

\section{Results}

Model description. Consider the Cooperative Decision Making model (CDMM) consisting of two-state units $s_{i}$, each of which represents an agent making decision to agree ("yes") or disagree ("no") on a given issue and whose dynamics are described by a master equation on a two-dimensional lattice ${ }^{15,16}$. In the absence of interactions the probability for a given unit to change its decision from "yes" to "no", or vice versa, is given by a Poisson distribution with a transition rate $g<1$. When the interaction between agents is turned on, the probability that a given unit is going to change its decision becomes time dependent, yielding transition rates

$$
p_{i \rightarrow j}(t)=g \exp \left[K\left\{M_{i}(t)-M_{j}(t)\right\} / M\right]
$$

where $M_{i}$ is the number of nearest neighbours in the state $i=$ " "yes", "no"\}, and $M$ is the total number of nearest neighbours. Global decisions of a network composed of $N$ units whose interactions are described by Eq. (1) can be defined by the time-dependent global order parameter

$$
\xi(t)=\left[N_{y e s}(t)-N_{n o}(t)\right] / N
$$

where $N_{\text {yes }}$ and $N_{n o}$ are the global counts of units being in one of the two states at a given time $t$. Here we consider the units of the CDMM to be on the nodes of a two-dimensional lattice with periodic boundary conditions. In the simulation each element is updated at each time step after which the transition rates in Eq. (1) are updated for the next time step.

Phase transition. The CDMM is similar to the well-known model proposed by Vicsek ${ }^{18}$ to study the cooperative dynamics of swarms of birds and demonstrates the shift from a configuration dominated by randomness to an organized state once the control parameter is increased above the critical value $K_{c}^{16}$. For values of the control parameter $K$ corresponding to the disorganized phase $K<K_{c}$, single units are only weakly influenced by the decisions of their neighbours and change their state with probability only slightly faster than the decoupled rate $g$. Thus, the fluctuations of the global order parameter $\xi(t)$ are characterized by small amplitude and very fast oscillations about the zero-axis [see Fig. 1a]. For $K>$ $K_{c}$, the interaction between units gives rise to a majority or consensus state, during which a significant number of agents adopt the same opinion at the same time [see Fig. 1c]. Figure 1d depicts the CDMM phase transition under the condition of nearest neighbour coupling on the two-dimensional lattice, where the time average of the global order parameter, $\xi_{\text {eq }}=\langle|\xi(t)|\rangle$, is used as a measure of the organization of the system.

We quantify the changes in temporal properties of the global variable $\xi(t)$, which accompany the phase transition, by evaluating the waiting-time probability density function $\Psi(\tau)$ and survival probability $^{16}$

$$
\Psi(\tau)=\int_{\tau}^{\infty} \psi\left(t^{\prime}\right) d t^{\prime}
$$

of time intervals $\tau$ between consecutive crossing of the zero-axis. As illustrated in Fig. 1e, in the subcritical regime $\Psi(\tau)$ has an exponential form, which reflects the large independence of single units from their neighbours. In the supercritical region $K>K_{c}$, the abrupt transitions between consecutive majority intervals, that result from the lattice having finite size, are responsible for an exponential shoulder present in $\Psi(\tau)$.

It is important to notice that for a given value of the transition rate $g$, departing significantly from the limiting condition $g \rightarrow 0$, there is no theoretical prediction for the critical coupling strength $K_{c}{ }^{16}$. The value of $K_{c}$ estimated with the traditional Binder cummulant method ${ }^{19}$ yields $K_{C}^{B}=1.644$ when $g=0.10$. However, since this approach estimates critical coupling for a network of infinite size, it is not surprising that $\Psi(\tau)$ evaluated for $K_{C}^{B}$ and a lattice of size $N=100 \times 100$ nodes shows an exponential shoulder, which is the hallmark of the organized phase. Simultaneously, following Vanni et al. ${ }^{20}$ we observe that for a network of finite size one can always find a value of the control parameter $K$ for which the exponential shoulder vanishes and for which $\Psi(\tau)$ is an inverse power-law (IPL) function. We assume this condition to correspond to the critical point for a network of finite size, and we obtain $K_{c}=1.625$ for $N=100 \times 100$ and $g=0.10$.

Critical exponents. The CDMM, defined by means of a two-state master equation, in which the transition rates between states depend on the local configuration of the lattice, has a number of properties in common with the kinetic Ising model ${ }^{21}$, where the dynamics of a 

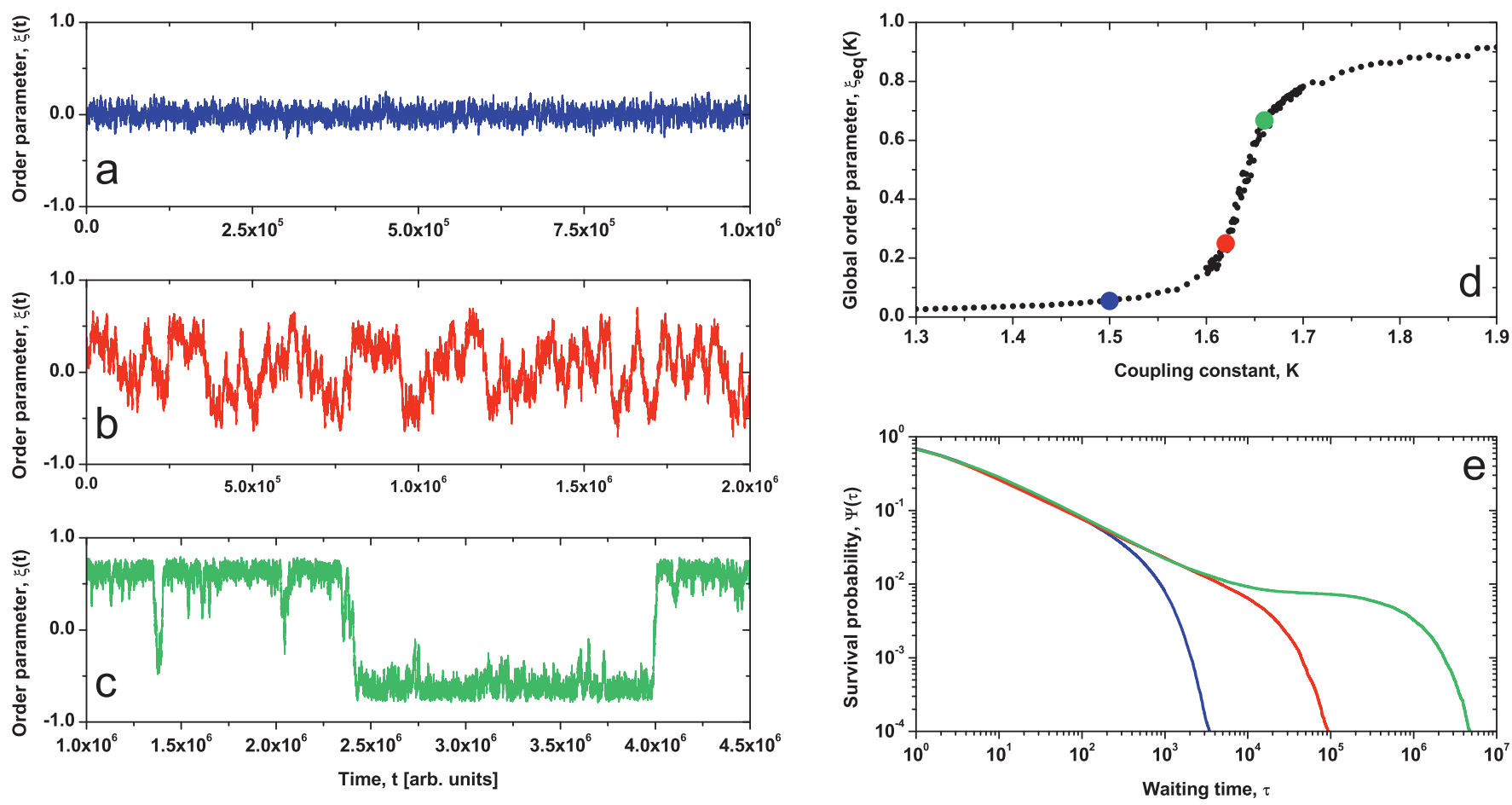

Figure $1 \mid(\mathrm{a}-\mathrm{c})$ Temporal evolution of the global order parameter for increasing values of the control parameter, $K=1.50, K=1.62$ and $K=1.66$. (d) Phase transition diagram for the amplitude of the global order parameter $\xi(t)$ as a function of the control parameter $K$. (e) Survival probability distribution $\Psi(\tau)$ for selected values of $K$ : blue line corresponds to $K=1.50$, red line to $K=1.62$ and green line to $K=1.66$. Lattice size is $N=100 \times 100$ nodes, and transition rate $g=0.10$; all the calculation were done with periodic boundary conditions.

single spin is a function of the temperature of a thermal bath in which system is submerged. If one disregards the physical aspect of temperature and other physical quantities that follow, then a correspondence can be found ${ }^{16}$ between the CDMM control parameter and the temperature which defines the organization in the kinetic Ising model. From this perspective the CDMM is a generalization of the dynamic phase transition models and as such it shares their scaling behaviour. To illustrate this fact we study scaling properties of the global variable. Fig. 2 a demonstrates that the $\xi_{\text {eq }}$ dependence on the control parameter rescales and therefore collapses onto a single curve for a system of increasing size, with exponents $\beta=1 / 8$ and $v=1$. Next, the exponent $\beta=1 / 8$ reappears in the scaling of $\xi_{\text {eq }}$ near the critical point (Fig. 2b):

$$
\xi_{e q}=\left(1-K / K_{C}\right)^{\beta} \text {. }
$$

Finally, the susceptibility,

$$
\chi=\left\langle\xi_{e q}^{2}\right\rangle-\left\langle\xi_{e q}\right\rangle^{2}
$$

scales as

$$
\chi \propto\left(\left|K-K_{C}\right| / K_{C}\right)^{-\gamma}
$$

with exponent $\gamma=7 / 4$, demonstrating that the CDMM belongs to the Ising universality class ${ }^{22}$. However, as pointed in earlier publications on $\mathrm{CDMM}^{15,16}$, the correspondence between the two models arises merely from their mathematical implementation, and their physical interpretations are fundamentally different. In the kinetic Ising model, the fluctuations are induced by a thermal bath, whereas in the CDMM variability arises from within the system itself, as a consequence of its finite size. We noted previously that Grinstein et al. ${ }^{17}$ anticipated that such two-state probabilistic lattice models with local interactions would have dynamics belonging to the Ising universality class regardless of the source of fluctuations.

Three-dimensional CDMM. We observe the phenomenon of global cooperation introduced by the interactions between the units of the
CDMM in systems of lower and higher dimension than the regular two-dimensional lattice previously discussed. The mean-field approach is studied extensively in Turalska et al. ${ }^{15}$, where both phase transition and temporal complexity of the global variable are reported. Similar behaviour occurs in scale-free and random networks, as well as for dynamics on a three dimensional lattice. The latter case is illustrated in Fig. 3, where we show that the transition from lower to higher dimension does not significantly affect the qualitative behaviour of the model. First, we still perceive phase transition behaviour of the global variable. Next, the fluctuations of $\xi(t)$ increase in amplitude similarly to the behaviour observed in the twodimensional case. In the organized phase one observes time intervals with a majority of the units within the cube being in the same state. Finally, the distribution of those time intervals shows three distinct regimes, which were also present on the two-dimensional lattice.

Correlation function. Further insight into the dynamic properties of the CDMM is obtained by adopting the statistical measure of spatial influence, that being the correlation function ${ }^{23} C(r)$ between the nodes separated by the Euclidean distance $r$ :

$$
C(r)=\left\langle s_{i} s_{i+r}\right\rangle-\left\langle s_{i}\right\rangle\left\langle s_{i+r}\right\rangle
$$

The quantity $\left\langle s_{i} s_{i+r}\right\rangle$ denotes an average over all pairs of units on the lattice separated by distance $r$ and $\left\langle s_{i}\right\rangle=\left\langle s_{i+r}\right\rangle$ denotes averages over all the units of the network. Figure $2 \mathrm{c}$ shows $C(r)$ for selected values of the coupling constant $K$. For both sub-critical $\left(K<K_{c}\right)$ and supercritical $\left(K>K_{c}\right)$ values of the control parameter, the correlation function $C(r)$ decreases rapidly as a function of the distance between nodes. However, at criticality, $K=K_{c}$, we observe the correlation length to be significantly more extended than in either the sub- or super-critical regimes, a characteristic property of systems at a phase transition ${ }^{24}$. It is important to note that since we consider a network of finite size, this extended correlation implies the emergence of dynamical coupling between units that are not nearest neighbours and therefore not directly linked. 

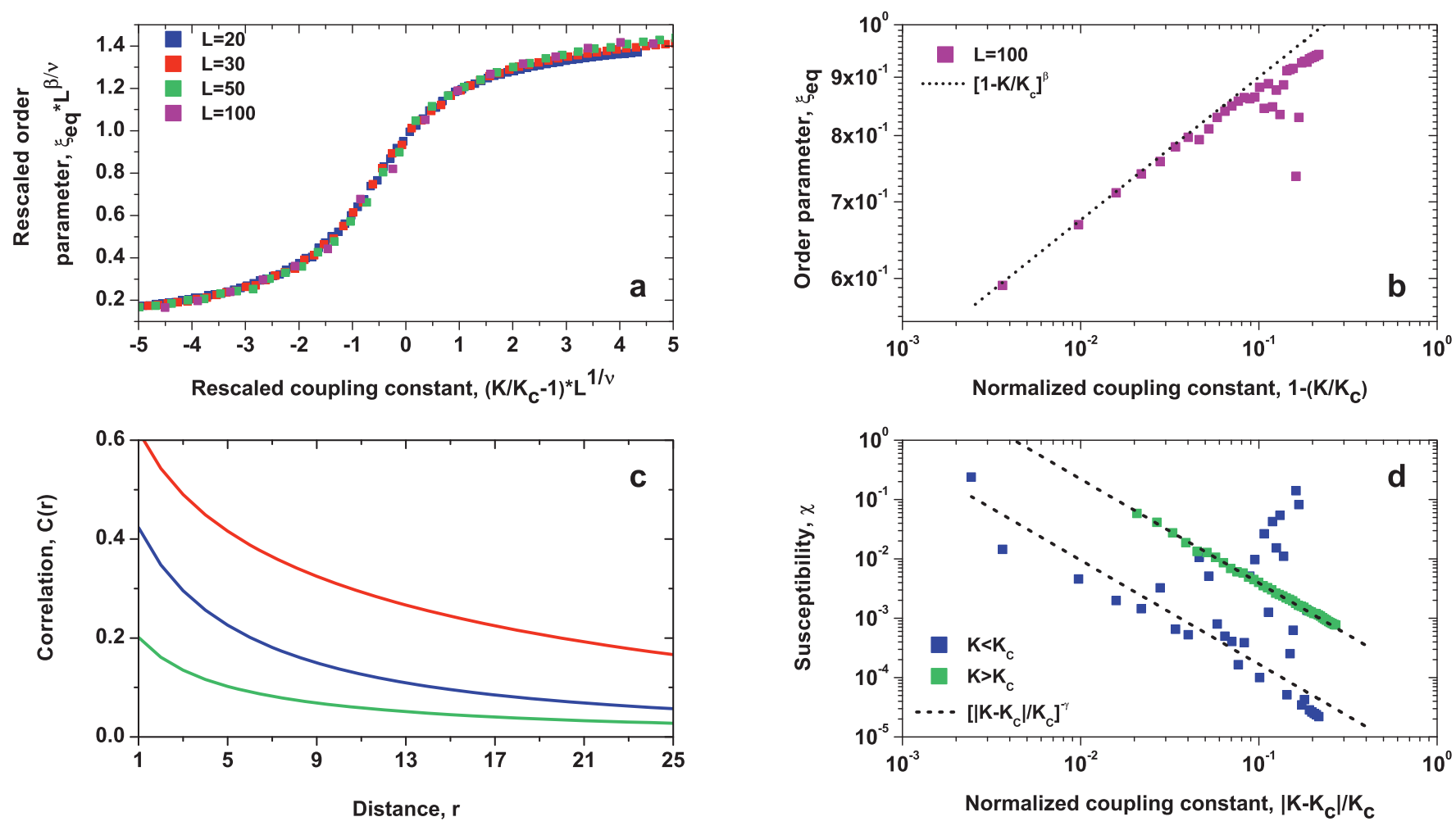

Figure $2 \mid$ Scaling properties of the CDMM. (a) Global order parameter $\xi_{e q}$ is evaluated as a function of the coupling constant for increasing size of the lattice and data collapse is observed for rescaled variables. (b) Scaling of the global order parameter in the vicinity of the phase transition point. (c) Correlation $C(r)$ as a function of the Euclidean distance $r$ between nodes of the lattice for $K$ being $K=1.50$ (blue line), 1.62 (red line) and 1.66 (green line), respectively. (d) Scaling of the susceptibility in the vicinity of the critical point. Critical control parameter $K_{c}=1.644, \beta=1 / 8, \gamma=7 / 4$ and $v=1$. Lattice size is $N=100 \times 100$ nodes, transition rate $g=0.10$.
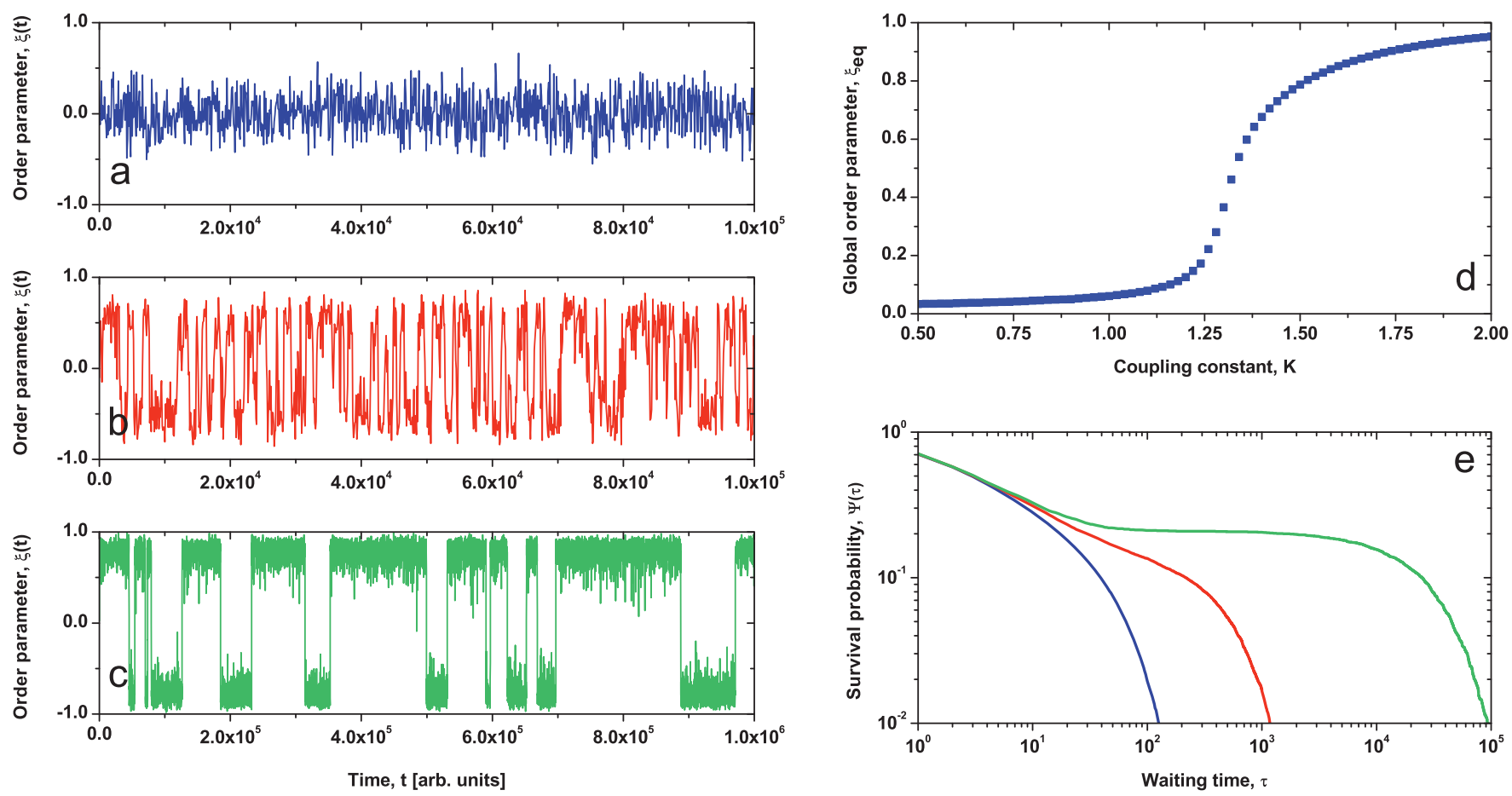

Figure 3 Behaviour of the CDMM on a three-dimensional simple cubic lattice. (a-c) Temporal evolution of the global order parameter for increasing values of the coupling constant, $K=1.00, K=1.30$ and $K=1.50$, respectively. (d) Phase transition curves for the CDMM evaluated on the 3D lattice of size $N=L^{3}$, for three sizes of the cube. (e) Survival probability function derived from the fluctuations of the global order parameter for increasing values of the coupling constant. Plots $(\mathrm{a}-\mathrm{c})$ and (e) refer to lattice of size $L=5$, plot (d) to lattice of size $L=10$ and for all simulations periodic boundary conditions were used, and $g=0.10$. 

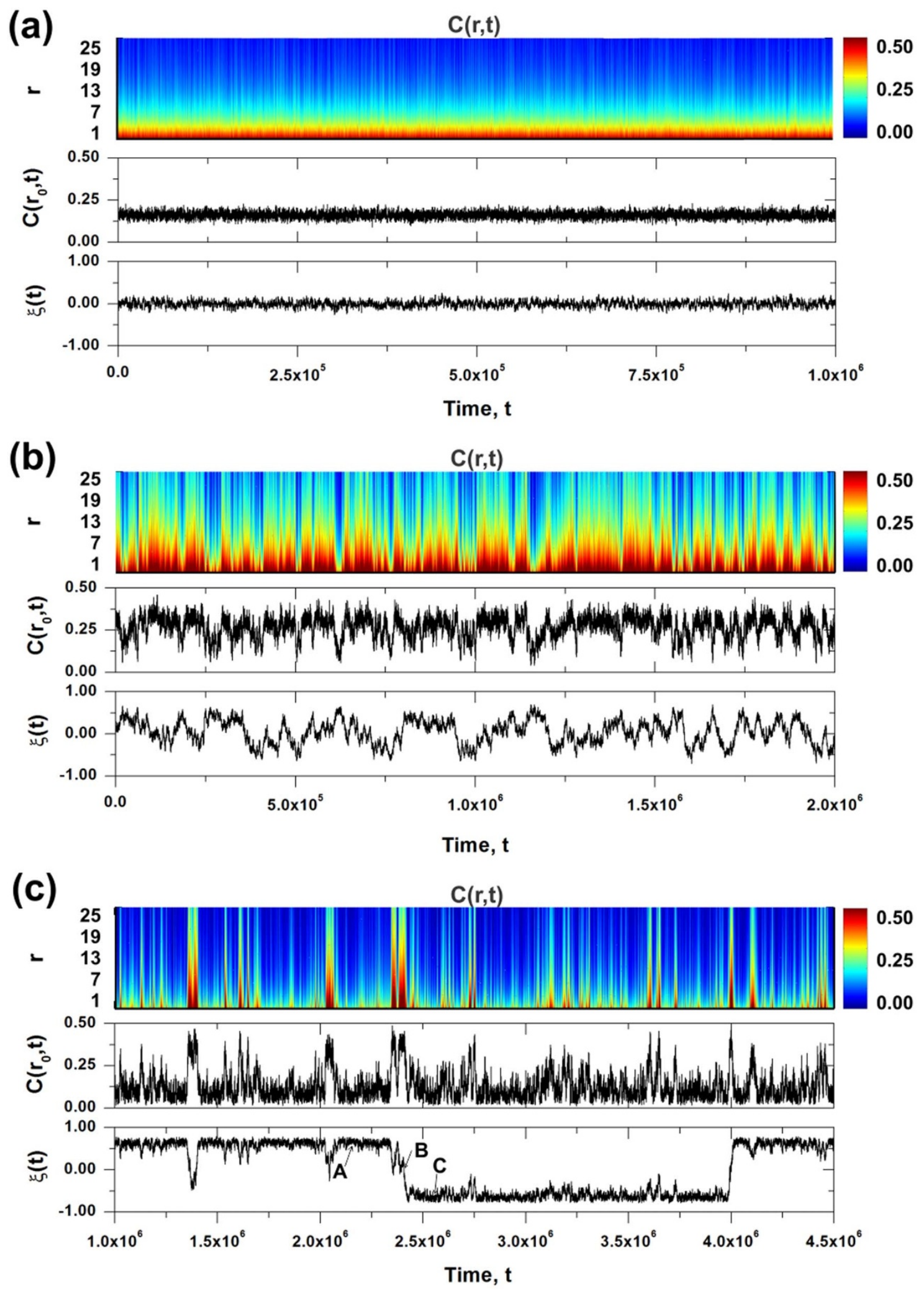

Figure $4 \mid$ Temporal evolution of the correlation function $C(r, t)$. On each panel color map encodes spatiotemporal variability of correlation $C(r, t)$. The middle plot shows cross-section of this map for $r_{0}=15$ and bottom plot shows corresponding evolution of the global order parameter $\xi(t)$. Analysis performed for $K=1.50$ (a), $K=1.62$ (b) and $K=1.66$ (c). Lattice size is $N=100 \times 100$ nodes and transition rate $g=0.10$.

Although traditionally the correlation function $C(r)$ is considered an equilibrium property of a network ${ }^{24}$, the central result of this paper is obtained by modifying Eq. (7) in order to study temporal fluctuations of the correlation function. For each instant of time we define the spatiotemporal correlation function

$$
C(r, t)=\left\langle s_{i}(t) s_{i+r}(t)\right\rangle-\left\langle s_{i}(t)\right\rangle\left\langle s_{i+r}(t)\right\rangle
$$

and as shown on Fig. 4, we observe that the fluctuations of the correlation function $C(r, t)$ closely track those of the global order parameter $\xi(t)$. In particular, the abrupt transitions between epochs 
a

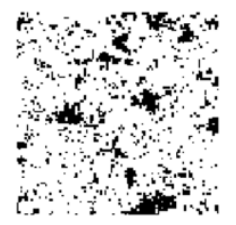

b

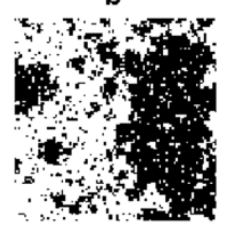

C

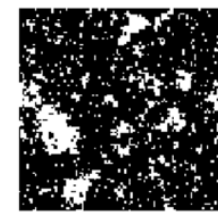

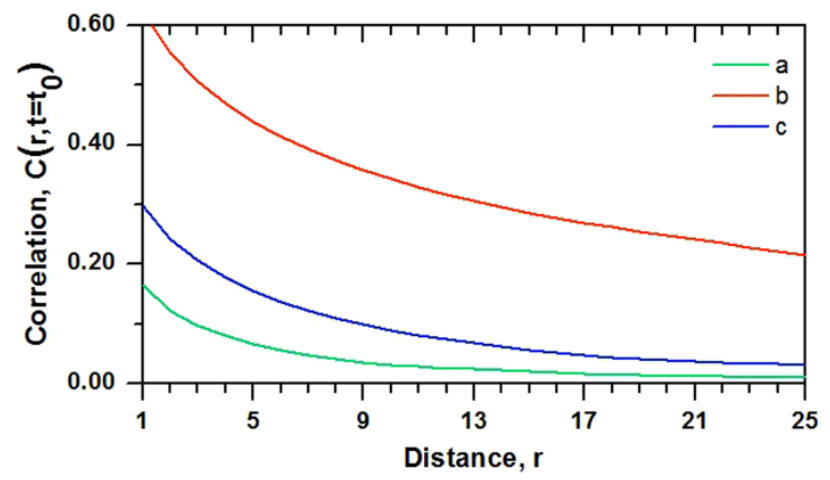

Figure $5 \mid$ Configuration of the lattice (a-c) and corresponding correlation function (bottom panel) for instances marked on bottom panel of Fig.4(c) by arrows. White areas correspond to the units in state "yes" and black to the units in the state "no". Lattice size is $N=100 \times 100$, coupling constant is $K=1.66$ and transition rate is $g=0.10$.

of dominating majority, the instances of crisis that take place when $K>K_{c}$, correspond to jumps in $C(r, t)$. Additionally, as demonstrated in Fig. 5, careful inspection of the network at the instant of crisis reveals an extended correlation length when compared with the organization of the lattice during majority rule. This observation provides an explanation of how committed minorities succeed in their goal of inducing significant social change. Since at the moment of the jump the order parameter vanishes, $\xi(t)=0$, we interpret those
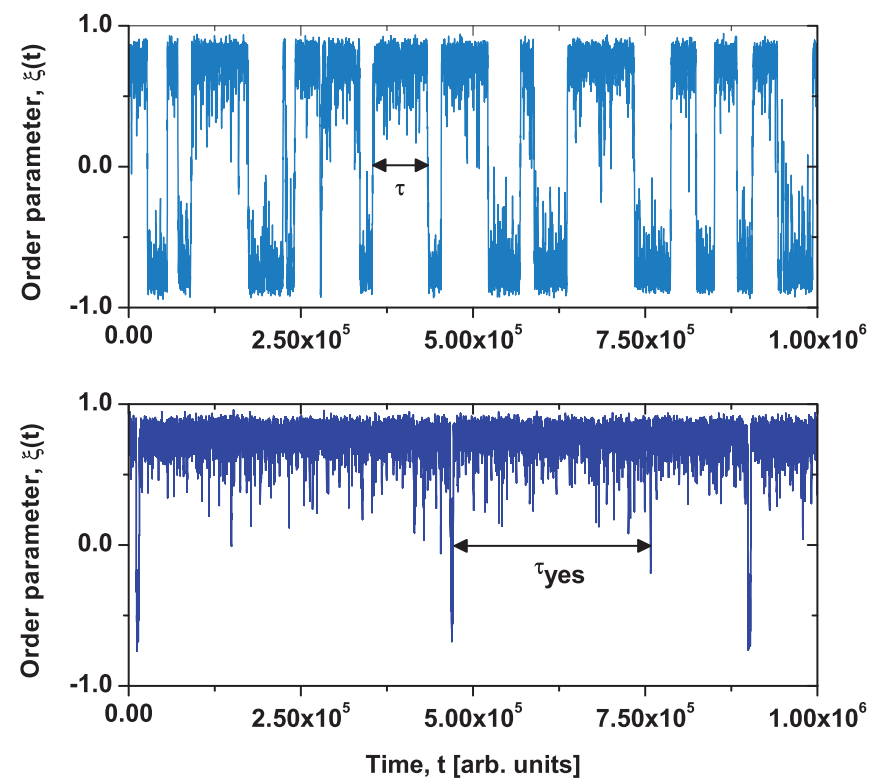

events as free will states. One could expect that the network had lost its organization and is randomly configured at that moment. However, such an interpretation would signify the presence of only local coupling and therefore could not explain how a small bias exerted on the network is able to produce large-scale changes, as shown subsequently.

Committed minority. A member of the committed minority considered herein is a randomly selected element on the lattice that keeps its decision of either "yes" or "no" independently of the opinion of its neighbours. To prove that the committed minorities may operate efficiently in spite of their very small number, in Fig. 6 we compare the evolution of $\xi(t)$ in the absence of a committed minority to the evolution of $\xi(t)$ in the presence of a relatively small (1\%) committed group. As might be expected the effect is observed both in the two and three dimensional system, letting us believe that it will also be present in networks of more complex topology. In the twodimensional case considered here a high value of the control parameter, $\left(K>K_{c}\right.$ ), leads to the extended condition of global consensus, during which the influence of the minority is negligible. The rapidly decreasing correlation function $C(r)$ reflects the rigidity of the network and prevents the global transmission of the perturbation. However, from time to time a crisis occurs where $\xi(t)=0$. In crisis the network may undergo an abrupt change of opinion and the correlation length is sufficiently large to make it possible for the committed minority to force the social network to adopt their view. As a consequence, during the time interval over which the minority acts it imposes its opinion over the whole network.

Finally, to quantify this phenomenon, we study how introducing a committed minority affects the average lifetime of the consensus state, with the average lifetime defined as

$$
\langle\tau\rangle=\int_{0}^{\infty} \Psi(\tau) d \tau
$$

It is important to note that in the condition where no committed group is present the distribution of time durations of global decision
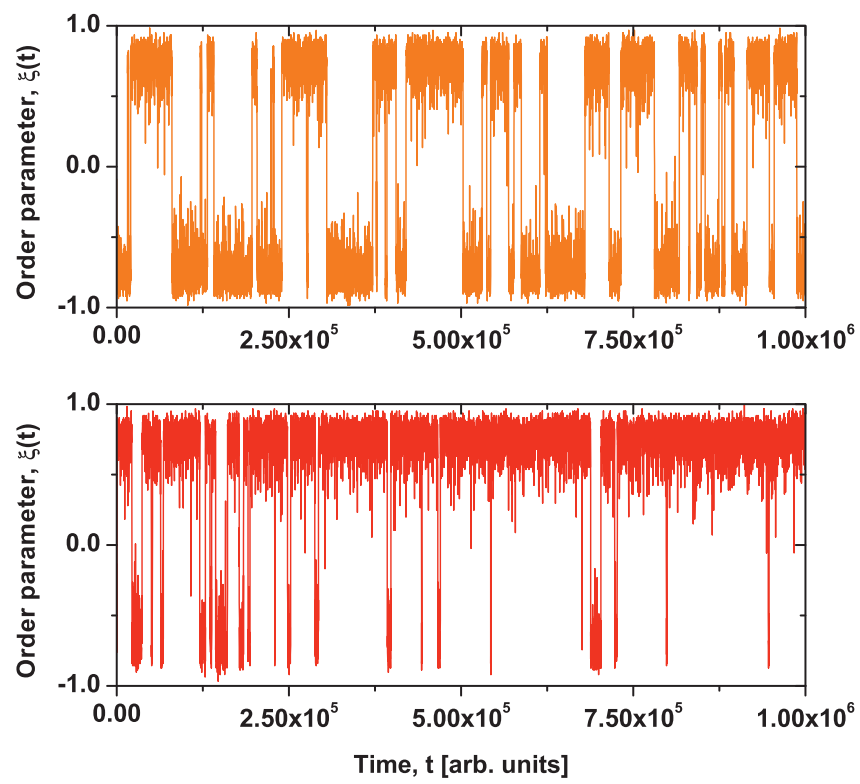

Figure 6 Temporal evolution for the global order parameter in the absence of the minority is compared with its evolution once a committed minority is present. (Left) Fluctuations of the global variable $\xi(t)$ for $K=1.65$ and the $2 \mathrm{D}$ lattice of size $N=100 \times 100$ nodes are compared with the behaviour of $\xi(t)$ once $1 \%$ of the randomly selected nodes are kept in state "yes" at all time. (Right) The dynamics of the CDMM on the 3D lattice when a $1 \%$ committed minority is present. The studied lattice has $N=5 \times 5 \times 5$ nodes, thus 1 node was always kept in state " +1 " to realize the committed minority concept. Coupling constant is $\mathrm{K}=1.40$. In both cases transition rate is $g=0.10$. Time interval $\tau$ marked on the top left panel denotes one of the global majority states. Its duration becomes significantly extended once committed minority is present, as can be seen on the bottom left panel. The time interval $\tau_{y e s}$ shows strong shift in the length of positive and negative majority states due to the presence of the minority. 

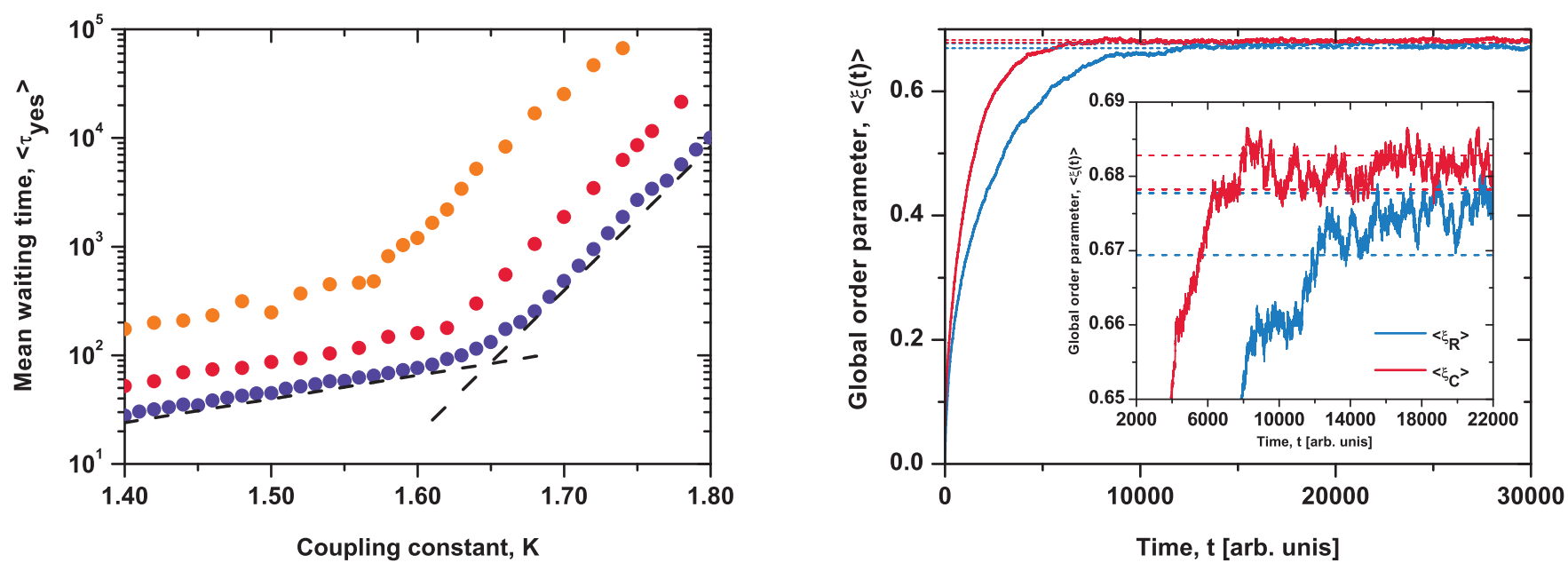

Figure $7 \mid$ (Left) Mean waiting time $\left\langle\tau_{\text {yes }}\right\rangle$ obtained for system with no acting minorities (blue dots) increases once 1\% (red dots) and 5\% (orange dots) committed minority is present. (Right) The ensemble average of 1000 independent realizations of the CDMM initialized with a random configuration $\left\langle\xi_{R}\right\rangle$ (blue line) is compared with those initialized with a crisis configuration $\left\langle\xi_{C}\right\rangle$ (red curve). Dashed lines correspond to the average value of the ensemble average in the long time limit with one standard deviation of its fluctuations added and subtracted.

in "yes", $\Psi\left(\tau_{\text {yes }}\right)$, and in "no", $\Psi\left(\tau_{n o}\right)$, coincide and are equal to the distribution evaluated for both time intervals, $\Psi(\tau)=\Psi\left(\tau_{\text {yes }}\right)=$ $\Psi\left(\tau_{n o}\right)$. This symmetry is however broken once a minority is introduced, as shown on Fig. 6, by an increase in temporal span of consensus states that correspond to the opinion of the minority. In Fig. 7 we compare $\langle\tau\rangle$ in the absence of the inflexible agents to $\langle\tau\rangle$ in the presence of a committed minority of sizes $1 \%$ and $5 \%$. First, in the native case the average consensus time $\langle\tau\rangle$ increases exponentially with an increase in the coupling strength $K$, showing a faster rise once $K>K_{c}$, with a discontinuous change at the critical interaction strength. This switch in the rate of increase confirms the validity of the approach used to determine $K_{c}$ based on the temporal properties of $\xi(t)$. Consecutively, the introduction of a small minority leads to a linear increase in $\langle\tau\rangle$ and the fact that two exponential regimes are preserved confirms the crucial role that instances of crisis play in the global transmission of minority opinion. Analogous behaviour is observed for the CDMM studied on a three-dimensional lattice. Note that this result differs from the transition found by Singh et al. ${ }^{14}$ from exponential to logarithmic dependence on network size at a critical value of the fraction of the committed minority. We attribute this functional change that is not observed in CDMM to the mechanism of dynamically rewiring the network introduced into their model by Singh et al. ${ }^{14}$

To provide additional evidence demonstrating the role of longrange correlations present in the network during the transition from one majority interval to another, we consider an ensemble of realizations of the CDMM process: one initialized with random configuration of the lattice and the other with a configuration derived from the instance of a crisis. Both ensembles of realizations of the CDMM process include $1 \%$ of committed nodes. This approach lets us investigate the influence of an initial condition on the evolution of stochastic realizations of the model. Due to the presence of a small minority each evolution of $\xi(t)$ spends more time in the state "yes" and gives rise to a non-zero ensemble average $\langle\xi(t)\rangle$. As shown on the Fig. 7, the ensemble average initialized with the state of crisis reaches an equilibrium earlier that the system randomly initialized. Since all other parameters of the dynamics are the same, this allows us to conclude that the long range correlations present in the system at the time of crisis do play a role in the spread of the opinion of the committed minority.

\section{Discussion}

Our approach does not allow us to test the speculation made by Xie et al. ${ }^{9}$ that the minimal size of the committed minority necessary to significantly affect the opinion of the entire network is $10 \%$. If we assume that a substantial effect of a committed minority is defined by an order of magnitude increase in the average consensus time $\langle\tau\rangle$, Fig. $7 \mathrm{a}$ indicates that for CDMM this requirement is realized by a committed minority of less than $5 \%$. Simultaneously, we observe that the effect of the minority on the dynamics of the CDMM is preserved once the dimension of the lattice is increased from two to three, which is contrary to the results reported by Mobilia ${ }^{25}$. We are convinced that those differences are a consequence of the non-local interactions present in the CDMM and the local, diffusion-like behaviour shown by the models investigated by others ${ }^{9,25}$.

The fact that CDMM generates consensus through a second-order phase-transition process is of crucial importance to the influence the committed minority has on the dynamics of the network. Due to the finite size of the network, the consensus states are transient, contrary to what is observed in the voter model or Naming Games, where the dynamics progress to an absorption-like global agreement state. The presence of a committed fraction of elements extends the global states of overlapping opinion; however it does not change their intermittent nature. Surprisingly, the instances at which the organization of the network disappears are indicative of the critical state, rather than being purely disordered in nature. The extended correlations present in the network at times of crisis facilitate the transmission of the opinion of the committed minority, providing a social mechanism for such an information transport process. This issue of facilitating opinion transfer is not discussed in earlier works on the role of zealots in networks.

Herein we have demonstrated that in a dynamical network the extended correlation length emerges not only at criticality, but quite surprisingly also at times of crisis which separate intervals of strong opinion. Our results indicate that this property is responsible for the significant efficiency of inflexible minorities in influencing entire networks. This discovery may shed light on the animal behaviour recently observed ${ }^{26}$ in flocks of locust or schools of fish and reproduced with the cooperative model of Vicsek ${ }^{18}$. In fact, the locusts' change of direction seems to occur in the supercritical condition and is accompanied by increased activity of the single insect. Simultaneously, it follows that there is no need for specific organization of the members of the committed minority, since due to increased correlation their impact is perceived even if the inflexible units are randomly arranged within the network and are not in direct contact with each other. 


\section{Methods}

The Cooperative Decision Making model (CDMM) consists of a system of $\mathrm{N}$ two-state units located at the nodes of a two and three dimensional square lattice. Each unit $s_{i}$ is a stochastic oscillator and can assume either of two states, +1 or -1 . The dynamics is introduced by choosing a single unit on a site $i$ and updating it in an elementary time step with a transition rate defined by Equation (1). Due to selected topology, the number of nearest neighbors is $M=4$ and $M=6$ for the square and cubic lattice, respectively. All numerical calculations were performed assuming periodic boundary conditions. In the case of the three-dimensional lattice the opposite facets of the cube were considered to be in direct contact. Initially the states of the single units were randomly assigned. Next, the dynamics of the model was repetitively evaluated at the consecutive time steps. In a single time step a run over the whole lattice was performed in a sequential fashion and for every unit $s_{i}$ the transition rate of Eq. (1) was calculated according to which a node was given the possibility to change its state. The size of the system was $N=100 \times 100$ for the square lattice and $N=5 \times 5 \times 5$ or $N=10 \times 10 \times 10$ in the case of the cubic lattice. In most cases the simulations of the CDM model were performed on a time scale of $10^{6}$ time steps for a selected value of the coupling constant $K$.

The time-dependent global order variable $\xi(t)$ is defined by equation (2). Its long-time scale properties are captured by the global order parameter, $\xi_{e q}=\langle|\xi(t)|\rangle$, which is calculated as a time average of the absolute value of $\xi(t)$ over the time window of $10^{6}$ time steps. The temporal properties of the global variable $\xi(t)$ are evaluated by the means of approximating the waiting-time probability density function $\Psi(\tau)$ with the normalized histogram of time intervals $\tau$ extracted from a single, long time scale evolution of the CDM process. Time intervals $\tau$ are defined as the intervals between the instances at which $\xi(t)$ changes sign, disregarding the amplitude of $\xi(t)$ or length of previous intervals $\tau$. In order to properly estimate $\Psi(\tau)$, the length of a CDM process needs to be increased from $10^{6}$ to $10^{8}$ as the dynamics moves from subcritical to supercritical regime and time intervals during which $\xi(t)$ keeps constant sign become larger and larger.

The correlation function $C(r)$ and its spatiotemporal equivalent $C(r, t)$, defined by equations (7) and (8), respectively, requires the calculation of an average over all pairs of units on the lattice separated by distance $r$. For simplicity of numerical calculations, we considered only the natural values of the separation distance $r$, disregarding possible contributions from elements separated in other manner. The calculation of the separation distance took into account periodic boundary conditions.

The committed minority concept was realized by selecting randomly a small fraction of the units of the system and freezing their state while letting other nodes follow the CDM dynamics as described earlier. Thus, committed nodes were allowed to influence their nearest neighbors, their state being included in the evaluation of the transition rate for those neighbors, while at the same time they disregarded any influence and kept their assigned state during the simulation time.

1. Handy, C. M. The Age of Unreason. (Harvard Business Review Press, 1991).

2. Ball, P. Crisis response: The new history. Nature 480, 447-448 (2011).

3. Vespignani, A. Complex networks: The fragility of interdependency. Nature 464, 984-985 (2010).

4. Barrat, A., Barthelemy, M. \& Vespignani, A. Dynamical processes on complex networks. (Cambridge University Press, 2008).

5. Haken, H. Cooperative phenomena in systems far from thermal equilibrium and in nonphysical systems. Rev. Mod. Phys. 47, 67-121 (1975).

6. Galam, S. \& Jacobs, F. The role of inflexible minorities in the breaking of democratic opinion dynamics. Physics A 381, 366-376 (2007).

7. Masuda, N. Evolution of cooperation driven by zealots. Sci Rep 2 (2012).

8. Mobilia, M., Petersen, A. \& Redner, S. On the role of zealotry in the voter model. J Stat Mech-Theory E 08, P08029 (2007).

9. Xie, J. et al. Social consensus through the influence of committed minorities. Phys. Rev. E 84, 011130 (2011).
10. Zhang, W., Lim, C. \& Szymanski, B. K. Analytic treatment of tipping points for social consensus in large random networks. Phys Rev E 86, 061134 (2012).

11. Halu, A., Zhao, K., Baronchelli, A. \& Bianconi, G. Connect and win: the role of social networks in political elections. arXiv, 1210.1996v1 (2012).

12. Marvel, S. A., Hong, H., Papush, A. \& Strogatz, S. H. Encouraging moderation: clues from a simple model of ideological conflict. Phys Rev Lett 109, 118702 (2012).

13. Couzin, I. D. et al. Uninformed individuals promote democratic consensus in animal groups. Science 334, 1578-1580 (2011).

14. Singh, P., Sreenivasan, S., Szymanski, B. K. \& Korniss, G. Accelerating consensus on coevolving networks: The effect of committed individuals. Phys Rev E 85, 046104 (2012).

15. Turalska, M., Lukovic, M., West, B. J. \& Grigolini, P. Complexity and synchronization. Phys Rev E 80, 021110 (2009).

16. Turalska, M., West, B. J. \& Grigolini, P. Temporal complexity of the order parameter at the phase transition. Phys Rev E 83, 061142 (2011).

17. Grinstein, G., Jayaprakash, C. \& Yu, H. Statistical mechanics of probabilistic cellular automata. Phys Rev Lett 55, 2527-2530 (1985).

18. Vicsek, T., Czirok, A., Ben-Jacob, E., Cohen, I. I. \& Shochet, O. Novel type of phase transition in a system of self-driven particles. Phys Rev Lett 75, 1226-1229 (1995).

19. Binder, K. Finite size scaling analysis of Ising model block distribution functions. $Z$ Phys B 43, 119-140 (1981).

20. Vanni, F., Lukovic, M. \& Grigolini, P. Criticality and transmission of information in a swarm of cooperative units. Phys Rev Lett 107, 078103 (2011).

21. Odor, G. Universality classes in nonequilibrium lattice systems. PRev Mod Phys 76, 663-724 (2004).

22. Christensen, K. \& Moloney, N. Complexity and Criticality. (Imperial College Press, 2005).

23. Wu, T. T., McCoy, B. M., Tracy, C. A. \& Barouch, E. Spin-spin correlation functions for the two-dimensional Ising model: Exact theory in the scaling region. Phys. Rev. B. 13, 316-374 (1976).

24. Fisher, M. E. The theory of equilibrium critical phenomena. Rep Prog Phys 30, 615 (1967).

25. Mobilia, M. Does a single zealot affect an infinite group of voters? Phys Rev Lett 91, 028701 (2003).

26. Yates, C. A. et al. Inherent noise can facilitate coherence in collective swarm motion. Proc Nat Acad Sci USA 106, 5464-5469 (2009).

\section{Acknowledgements}

MT and PG gratefully acknowledge ARO for the financial support of this research through grant W911NF-11-1-0478.

\section{Author contributions}

M.T. \& P.G. designed research. M.T. performed simulations. M.T., P.G. \& B.W. analyzed the data. All authors wrote, reviewed and approved the manuscript.

\section{Additional information}

Competing financial interests: The authors declare no competing financial interests.

License: This work is licensed under a Creative Commons

Attribution-NonCommercial-ShareALike 3.0 Unported License. To view a copy of this license, visit http://creativecommons.org/licenses/by-nc-sa/3.0/

How to cite this article: Turalska, M., West, B.J. \& Grigolini, P. Role of committed minorities in times of crisis. Sci. Rep. 3, 1371; DOI:10.1038/srep01371 (2013). 